\title{
Changes of cytosine methylation in pecan tissues of different stages by quantitative methylation-sensitive amplified polymorphism
}

\author{
Z.Z. $\mathrm{LIU}^{1}$, F. ZHOU${ }^{1}$, J. SHANG ${ }^{1}$, F.R. PENG ${ }^{1 *}$, Z.H. MO², and Y.R. LI ${ }^{3}$ \\ College of Forestry and Co-Innovation Center for the Sustainable Forestry in Southern China, \\ Nanjing Forestry University, Nanjing 210037, P.R. China ${ }^{1}$ \\ Institute of Botany, Jiangsu Province and Chinese Academy of Sciences, Nanjing 210014, P.R. China ${ }^{2}$ \\ Green Universe Pecan Science and Technology Co., Nanjing 210007, P.R. China ${ }^{3}$
}

\begin{abstract}
Cytosine methylation plays an important role in plant development by regulating gene expressions. However, few studies have investigated methylation changes during the tissue differentiation and development of perennial plants. Here, the fluorescence-labeled methylation-sensitive amplified polymorphism method was used with eight primer combinations to detect methylation in leaves and xylem obtained at the stages of inflorescence emergence (IE), ovary start growth, and fruit maturity (FM) in two pecan (Carya illinoinensis) cvs. Pawnee and Stuart. The results show that the total methylation in the xylem was generally higher than in the leaves at each stage. Substantial methylation variations were observed at the amplified sites in pecan tissues at the various stages. The methylation patterns changed between the leaf and xylem, with frequencies from 44.97 to $67.01 \%$ over the three stages in the two cultivars, among which the variation frequency between the tissues at the FM stage was the highest for each cultivar. The frequencies of methylation variation between the leaf samples at any two stages ranged from 31.86 to $45.88 \%$, with higher variation frequencies between the xylem samples (40.90 - 59.44 \%) for each cultivar, which is consistent with the comparative results of polymorphism rates between the leaf and xylem over the three stages. Cluster analysis and principal coordinate analysis suggest that the xylem at the IE and FM stages had relatively distant epigenetic relationships with other tissue samples as a whole. This study reveals the patterns of methylation variation and methylation relationships among pecan tissues undergoing different developmental processes, implying the important roles of methylation in tissue differentiation and development of trees. These results lay a theoretical foundation for elucidating the regulatory mechanisms of methylation involved in tree development.
\end{abstract}

Additional key words: Carya illinoinensis, fluorescence-labeled methylation-sensitive amplified polymorphism, plant development.

\section{Introduction}

DNA cytosine methylation (5-methylcytosine formation), a prominent epigenetic modification shared by a vast range of eukaryotic organisms, refers to the transfer of methyl groups from S-adenosyl-L-methionine to the fifth carbon of cytosine (Lu et al. 2008, Vining et al. 2012, Zhang et al. 2010). In plants, DNA methylation can occur in all cytosine sequence contexts: $\mathrm{CG}, \mathrm{CHG}$, and $\mathrm{CHH}(\mathrm{H}=\mathrm{A}, \mathrm{C}$, or $\mathrm{T})$ (He et al. 2011). The de novo methylation of cytosines is established primarily by the catalysis of domain rearranged methyltransferase 2 (DRM2) (Cao and Jacobsen 2002). Ribonucleic acid directed DNA methylation (RdDM) plays an important role in this process, in which the siRNA initiated by RNA polymerase IV guides the targeting of nascent scaffold transcripts from Pol V by sequence complementarity, and this targeting recruits DNA methyltransferase activity to mediate de novo methylation of cytosines (Matzke and Mosher 2014). CG, CHG, and $\mathrm{CHH}$ methylation is maintained by different mechanisms: CG methylation is maintained by methyltransferase 1 (MET1); CHG methylation is maintained by chromomethylase 3 (CMT3) and to a much lesser extent by CMT2; and CHH methylation is maintained by DRM2 or CMT2 (Zhang et al. 2018). Findings from Arabidopsis thaliana showed that extensive methylation occurs in

Submitted 5 October 2019, last revision 13 December 2019, accepted 7 January 2020.

Abbreviations: CMS - changes in pattern of methylation state; DM - demethylation; DNM - de novo methylation; FM - fruit maturity; F-MSAP - fluorescence-labeled methylation-sensitive amplified polymorphism; IE - inflorescence emergence; OSG - ovaries start growth; PCoA - principal coordinate analysis.

Acknowledgments: This research was supported by a grant from the National Key R\&D Program of China (2018YFD1000600), the National Natural Science Foundation of China (31870672), the Innovation and Promotion Project of Forestry Science and Technology of Jiangsu Province (LYKJ[2018]05-1), the Priority Academic Program Development of Jiangsu Higher Education Institutions (PAPD) and the Doctorate Fellowship Foundation of Nanjing Forestry University.

*Corresponding author; e-mail: frpeng@njfu.edu.cn 
transcriptionally inactive heterochromatic regions, which are densely packed with transposons and other repetitive sequences, and a considerable amount of methylation is also shown in euchromatic regions, including genes and nonrepetitive intergenic regions (Zhang et al. 2006). Cytosine methylation is shown as a stable epigenetic mark in most cases (Law and Jacobsen 2010), and little is known about the dynamic nature of DNA methylation. However, the dynamic changes in methylation in the plant genome have attracted attention from researchers, and one report on Arabidopsis has even suggested a highly variable distribution of cytosine methylation within plant genomes (Vaughn et al. 2007). It has been known that cytosine methylation plays an important role in the regulation of gene expression (Zhang et al. 2006), transposon silencing (Li et al. 2015), heterochromatin organization (Soppe et al. 2002), and genomic imprinting (Jullien et al. 2006).

Plant development is achieved through the spatial and temporal regulation of gene expression in a complex genetic network (Viejo et al. 2012). Cytosine methylation is known to play an important role in the regulation of plant development, and this was mainly revealed by use of the various loss-of-function methylation mutants (Zhang et al. 2010). For instance, a study using antisense MET1 plants of Arabidopsis reported that decreased methylation will result in a number of phenotypic and developmental abnormalities, such as smaller plant size, altered leaf size and shape, and altered flowering time (Finnegan et al. 1996). A study on Glycine max seed development showed that DNA methylation underwent dynamic changes during seed maturation, and $\mathrm{CHH}$ methylation in cotyledons changed greatly from $6 \%$ at the early stage to $11 \%$ at the late stage (An et al. 2017). Zhong et al. (2013) identified 52095 differentially methylated regions (representing $1 \%$ of the genome) during the development of Solanum lycopersicum. However, there is still a lack of evidence regarding the dynamic changes in methylation status across development, especially in perennial plants. Tissue differentiation and formation are important aspects of plant development, and tissue-specific variation in cytosine methylation has been identified in some plant species. For example, specific methylations were found in different tissues of Daucus carota (Palmgren et al. 1991) and S. lycopersicum (Messeguer et al. 1991). In Zea mays, different methylation patterns were observed in the tassel, bracteal leaf, and ear leaf (Lu et al. 2008). The results from Sorghum bicolor suggested that the endosperm was among tissue-specific methylated regions (TDMRs) and that more extensive demethylation was present in the endosperm than in other tissues (Zhang et al. 2011). Recently, in the model tree species Populus trichocarpa (Vining et al. 2012) and Populus tomentosa (Wang et al. 2016), tissuespecific methylation variation was revealed. However, the patterns of methylation changes between tissues still need to be further elucidated especially in perennial plants.

The methylation-sensitive amplified polymorphism (MSAP), a stable and relatively low-cost method to detect cytosine methylation, has been applied widelyin studies of methylation events during growth and development (Candaele et al. 2014), stress resistance (Wang et al. 2011), heterosis (Li et al. 2018), and biodiversity (Salmon et al. 2008). The MSAP technique was established based on amplified fragment length polymorphism (AFLP) analysis and depends on using two different DNA methylationsensitive restriction isoschizomers ( $H p a \mathrm{II}$ and $M s p \mathrm{I}$ ) for the same restriction site (CCGG) (Reyna-López et al. 1997). HpaII is inactive if one or both cytosines are methylated on both DNA strands but cleaves when external cytosine is methylated on only one strand, whereas MspI cleaves if internal cytosine is fully methylated but cannot cleave at an external methylated cytosine (Salmon et al. 2008, Zhao et al. 2015, Li et al. 2018). In traditional MSAP procedures, the bands are detected with polyacrylamide gel electrophoresis (PAGE). The fluorescence-labeled methylation-sensitive amplified polymorphism (F-MSAP) technique was introduced later, with capillary electrophoresis as the detection method (Xu et al. 2005). With the advantages of increased safety, efficiency, and resolution, the F-MSAP technique has shown good potential for broad application for methylation detection in plants in recent years (Herrera and Bazaga 2010, Inácio et al. 2017). Substantial methylation information at CCGG sites can be detected via F-MSAP or traditional MSAP techniques; however, it is not easy to analyze methylation events and extract valuable information. Although MSAP data have been analyzed in many studies, and some information has accumulated regarding the analysis of methylation events, the exploration of the establishment of quantitative methods for the complete interpretation of MSAP data has rarely been reported except that the relative quantitative MSAP method was creatively put forward for the analysis of abiotic stress (Bednarek et al. 2017).

Pecan (Carya illinoinensis) is an important nut crop originating from North America and has been widely planted in many countries worldwide. Pecan nuts are the major economic value of pecan trees, and thus, many studies have focused on fruit development, whereas minimal attention has been paid to the study of vegetative tissue or organ development. The vegetative organs are responsible for producing and transporting nutrients to trees, and their normal differentiation and development are crucial to the development and productivity of an entire tree. Therefore, it is also important to conduct research to explore the regulatory mechanisms in differentiation and development of vegetative tissues or organs in pecan trees. As an important regulator of plant development, cytosine methylation has not been studied in pecan apart from our previous report, in which F-MSAP procedures were used to study mainly the methylation variation among pecan cultivars and a preliminary analysis was performed to evaluate the methylation changes in leaves over developmental stages (Liu et al. 2018). With the aim to detect the methylation patterns at the CCGG sites of leaves and xylems at three developmental stages, the same F-MSAP procedure was used. The tissue materials at different ontogenetic stages were used as ideal templates to establish an MSAP analysis for the quantification of MSAP data. In addition, this study explored the differences in methylation between the leaf and xylem. This study can reveal the rules of methylation changes and epigenetic 
relationships among pecan tissues at different stages, which can serve as a theoretical foundation for further research on the regulatory mechanisms of methylation in tree development.

\section{Materials and methods}

Plants, cultivation, and sampling: Two broadly planted pecan [(Carya illinoinensis (Wangenh.) K. Koch] cultivars Pawnee and Stuart were selected for this study. 'Pawnee' originated from a cross of cvs. Mohawk and Starking Hardy Giant (Thompson and Hunter 1985), and 'Stuart' is of unknown parentage but cultivated approximately from 1874 (Lombardini et al. 2009). They were cultivated at Nanjing Green Universe Pecan Science \& Technology Co., Luhe district of Nanjing, Jiangsu Province, China at lat. $32^{\circ} 19^{\prime} 59.48^{\prime \prime} \mathrm{N}$, long. $118^{\circ} 52^{\prime} 22.37^{\prime \prime} \mathrm{E}$, and $170 \mathrm{~m}$ altitude. Three six-year-old trees (grafted on two-year-old rootstocks) were selected and the leaves and xylems were collected on 25 April, 25 May, and 28 September 2016, which were defined as the inflorescence emergence (IE), ovaries start growth (OSG), and fruit maturity (FM) stages, respectively, based on the Biologische Bundesanstalt, Bundessortenamt und Chemische Industrie scale for pecan (Han et al. 2018). A sharp blade was used to first strip the bark (approximately $2 \times 2 \mathrm{~cm}$ ) of the tree trunk at a height of $20-30 \mathrm{~cm}$ above the ground, and then, part of the xylem was extracted as an experimental material. The $2^{\text {nd }}$ to $4^{\text {th }}$ pairs of leaflets from the basal portion of the compound leaves growing in the middle of one-year-old southfacing shoots approximately $2 \mathrm{~m}$ above the ground were sampled. These leaf samples collected at the IE, OSG, and FM stages belonged to the young, half-mature, and mature leaves, respectively. The collected tissue samples were rapidly taken to the laboratory on ice and frozen at $-70^{\circ} \mathrm{C}$.

Extraction of DNA: Genomic DNA was extracted from the mixed tissue samples using the cetyltrimethylammonium bromide (CTAB) method as described previously (Liu et al. 2018). The isolated DNA was checked by agarose gel electrophoresis and quantified with a NanoDrop 2000 microvolume spectrophotometer (Thermo Fisher Scientific, Waltham, USA).

Detection of cytosine methylation: To detect genomic methylation in different tissue samples of pecan, F-MSAP procedures, including double digestion with restriction endonuclease combinations of EcoRI/HpaII and EcoRI/ MspI, ligation, preselective amplification, and selective amplification with eight primer combinations, were used (Table 1 Suppl.) as described in a previous study (Liu et al. 2018). The products of selective amplification were detected via capillary electrophoresis.

Data analysis and statistics: In this study, we summarize and establish an MSAP analysis process (Fig. 1) using the obtained tissues with the same genotype as templates and apply it to analyze the MSAP data of the tissues at different stages. As shown in Fig. 1, a 1/0 matrix was first scored from capillary electrophoresis or PAGE ( 1 and 0 refer to the presence and absence of a fragment, respectively). Then, four types of fragment patterns, I $(1,1)$, II $(1,0)$, III $(0,1)$, and IV $(0,0)$, were recorded. Type $I(1,1)$ indicated digestion with both enzymes at an unmethylated CCGG site. Type II $(1,0)$, representing digestion with EcoRI/ HpaII but not EcoRI/MspI, indicated the presence of hemimethylation at the external cytosine of one strand. Type III $(0,1)$, showing digestion with EcoRI/MspI but not EcoRI/HpaII, indicated full methylation at the internal cytosine of double strands. Type IV $(0,0)$ indicated the inhibition of digestion with both enzymes at a fully methylated site (hypermethylation, methylation at both external and internal cytosines) when other samples with the same genotype presented any one of the other three pattern types at the same site (Salmon et al. 2008, Zhao et al. 2014, Li et al. 2018); otherwise, it represented a mutation at the sites. Based on the statistics of the bands of four methylation types, the methylation levels were obtained with the following formulas (Liu et al. 2018): hemimethylation = type II bands / total bands; full methylation = type III bands / total bands; and hypermethylation = type IV bands / total bands (total bands = type I + type II + type III + type IV bands).

In this analysis process, the different methods were presented corresponding to the quantitative analysis of the methylation events in the different samples at one level and at different levels. The samples (e.g., tissues) indicate those that are treated under one factor, and the levels (e.g., stages) indicate another factor influencing the samples. For the samples at one level, the methylation events included the same patterns and polymorphic patterns. The same patterns were classified into two types: nonmethylation state preservation and no change in pattern of methylation state. Polymorphic patterns were defined as different patterns among samples, consisting of specific patterns and other patterns (for over two samples). The specific patterns were defined as the patterns in one sample that were different from the uniform patterns at the same sites of other samples. The patterns comprised three types: one type referring to the methylation in one sample with unmethylation in other samples (demethylation, DM); another type referring to unmethylation in one sample with methylation in other samples (de novo methylation, DNM); and the last type referring to one methylation pattern in one sample with one of the other two methylation patterns in other samples (changes in pattern of methylation state, CMS). When there are three or more samples at one level for analysis, the pairwise comparison can be performed combined with the overall analysis of these samples to fully understand the methylation changes among them. For the analysis of the methylation status at the sites of the samples throughout different levels, the methylation events were classified into different types, including the same patterns present among the samples at any level and polymorphic patterns present among the samples at one level, two (or more) levels and any level. From these types, special forms of methylation events were also separated, including the identical patterns present among all of the samples and the same specific patterns present between 
two samples among different levels.

For the different samples at one level or different levels, analytical methods such as cluster analysis and principal coordinate analysis ( $\mathrm{PCoA}$ ) could be conducted to analyze the epigenetic structure and relationships. It should be noted that before the cluster analysis and PCoA were conducted, the raw 1/0 matrix required transformation into the new $1 / 0$ matrix. There are basically three main groups of transform methods that have been reported (Schulz et al. 2013), including 'methylation scoring', 'non-methylation scoring' and 'mixed scoring'. Here, 'mixed scoring 2' (Schulz et al. 2013) was used as a reference to transform the raw matrix obtained from the pecan tissues. Notably, for tissue materials in the present study, type IV was confirmed as the hypermethylation status, and the matrix for hypermethylation was additionally included except for the matrixes for unmethylation, hemi-methylation, and full methylation (the transform method is shown in Table 2 Suppl.). NTSYS software (v. 2.10e; Applied Biostatistics, New York, USA) was used to perform the cluster analysis and PCoA based on the four types of binary matrixes, and the dendrogram and PCoA diagrams were produced from it. The analysis by the software was conducted as described in our previous report (Liu et al. 2018).

The bar graphs in this paper were drawn using SigmaPlot software (v. 12.5; Systat Software, Chicago, USA); pie charts were produced from Excel (version 2016; Microsoft, Redmond, USA), and heat maps were created online at the following website (https://software. broadinstitute.org/morpheus/). The drawing of the flow chart was completed in Adobe Illustrator CC (version 2017; Adobe, San Jose, USA).

\section{Results}

In this study, the MSAP analysis was established and applied to analyze the F-MSAP data from the leaves and xylems of two pecan cultivars (Pawnee and Stuart) at the IE, OSG and FM stages. Examples of detected methylation patterns obtained from capillary electrophoresis are shown in Fig. 1 Suppl. Through statistical analysis, a total of 885 sites were scored by eight pairs of EcoRI+HpaII/ $M s p$ I selective primer sets for each cultivar, and the total methylation ranged from $56.95 \%$ in the 'Pawnee' leaf at the OSG stage to $66.33 \%$ in the 'Pawnee' xylem at the FM stage. The total methylation of the leaves in the two cultivars (average: $58.76,58.42$, and $59.72 \%$ for the IE, OSG, and FM stages, respectively) were generally lower than those of the xylems at each stage (average: $61.47,62.99$, and $61.86 \%$ for the three respective stages, respectively). The average methylation of the leaves of the two cultivars decreased from the IE stage to the OSG stage and then increased at the FM stage, which was in contrast to the trend of methylation changes in the developmental process of the xylem. Statistical analyses revealed that the differences in the total methylation between the leaf and xylem varied with the different developmental stages, with values of $3.73,5.08$, and $5.88 \%$ for the IE, OSG, and FM stages of 'Pawnee', respectively, and of 1.69, 4.07, and $1.58 \%$ for the IE, OSG, and FM stages of 'Stuart', respectively. These results also indicated that the difference in the total methylation between the leaf and xylem at each stage was greater in 'Pawnee' than in 'Stuart'. The levels of the three methylation patterns were compared, and the hypermethylation level was the highest for each tissue from the two cultivars at each stage $(26.55-34.12 \%)$, while the hemi-methylation level was the lowest (11.30 $17.29 \%$ ) (Fig. 2).

Comparisons of tissues at each stage were conducted to explore the differences in methylation status of the CCGG sites between pecan leaf and xylem at each stage. The results showed that the total frequencies of methylation variation (sum of $\% \mathrm{DM}+\% \mathrm{DNM}+\% \mathrm{CMS}$ ) were 60.90 , 44.97, and 67.01 between the tissues at the IE, OSG, and FM stages of 'Pawnee', respectively, with variation frequencies of 51.98, 53.67, and $54.12 \%$ between the tissues at the three stages of 'Stuart', respectively. For both cultivars, the total variation frequency between the leaf and xylem was highest at the FM stage. The percentages of DM from the leaf to xylem ranged from 8.47 to $16.27 \%$ for the three stages of the two cultivars, with a range of 13.56 to $19.55 \%$ for DNM and 22.94 to $33.79 \%$ for CMS. Among the percentages of the three variation types, \%CMS was the highest, and \%DM was generally the lowest (Fig. 3).

To compare the methylation patterns between the leaf and xylem at the same sites of the tissues at the three stages, using the quantitative methods described in the MSAP analysis (Fig. 1), the methylation events can be classified into four classes (class A: the same patterns present in two tissues at any stage, class B: different patterns present in two tissues at one stage, class C: different patterns present in two tissues at two stages, and class D: different patterns present in two tissues at any stage). Statistically, the sites with class $\mathrm{C}$ had the highest proportion in both 'Pawnee' and 'Stuart' (39.89 and 32.32\%), followed by the sites with class B (29.60 and $27.68 \%$ ), class D (16.95 and $22.49 \%$ ), and class A (13.56 and $17.51 \%)$. Among the sites with class $\mathrm{D}$, there was a group of sites with different patterns between the leaf and xylem maintained at the three stages, which accounted for 1.02 and $1.58 \%$ of all detected sites in 'Pawnee' and 'Stuart', respectively (Fig. 4).

The changes in methylation over developmental stages were investigated via pairwise comparison of the samples of each tissue at the three stages (IE, OSG, and FM). The results showed that the total frequency of methylation variation between the leaf samples at any two stages ( 31.86 - $45.88 \%$ ) was less than that between the xylem samples (40.90 - $59.44 \%$ ) for each cultivar. Particularly, among the total variation frequencies, the lowest $(31.86 \%)$ and highest $(59.44 \%)$ methylation variation were observed in the leaf and xylem between the IE and OSG stages of 'Pawnee'. The ratios of DM between any two stages were $8.14-12.54 \%$ in the leaves and $7.91-17.51 \%$ in the xylems of the two cultivars; the percentages of DNM between any two stages were $7.80-14.80 \%$ in the leaves and $10.62-17.85 \%$ in the xylems, while $\%$ CMS was the highest among the three variation types present in each tissue between any two stages (15.25 - $21.36 \%$ in the leaves and $22.03-26.44 \%$ in the xylems) (Fig. 5). 
MSAP analysis process
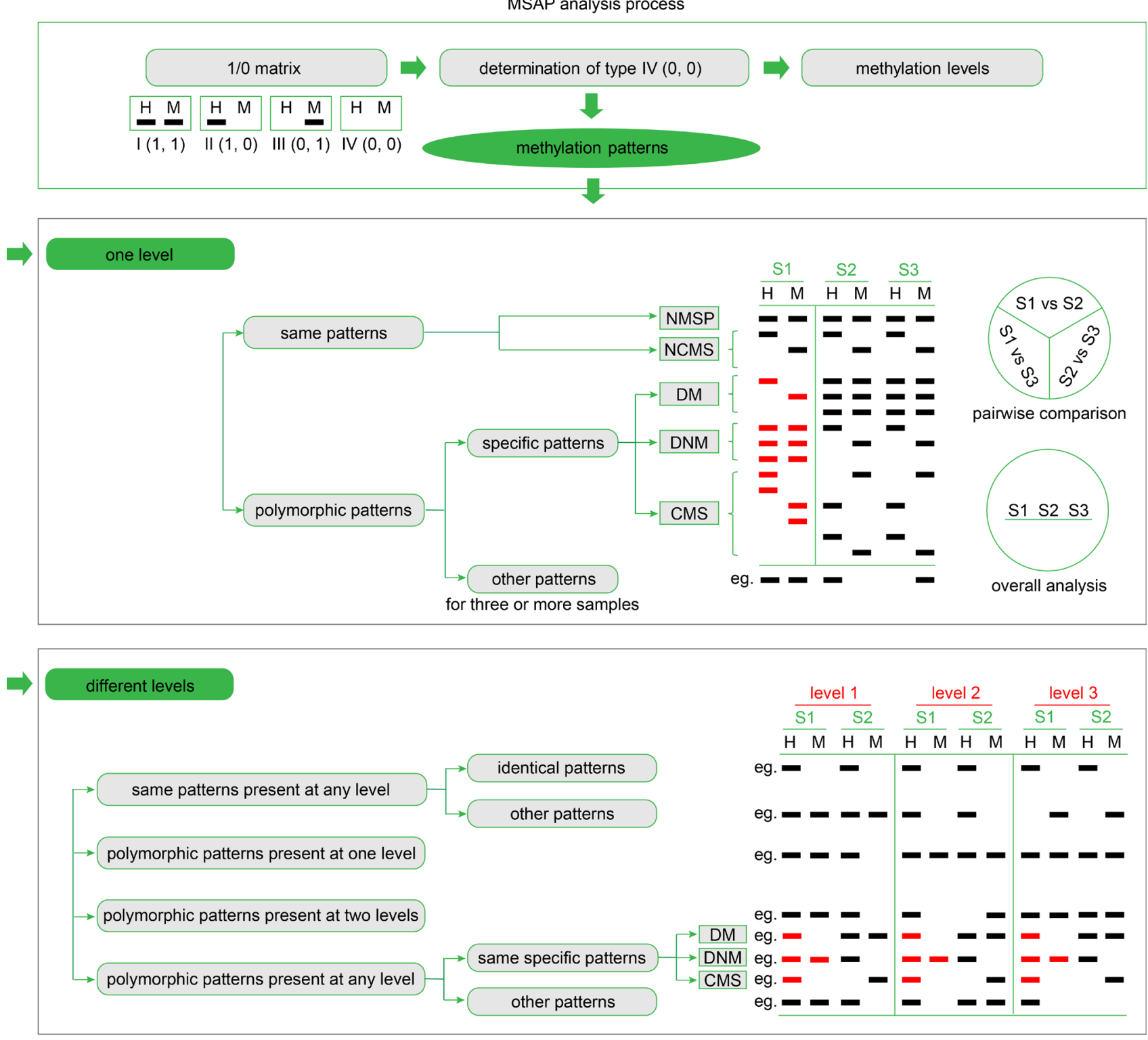

epigenetic structure

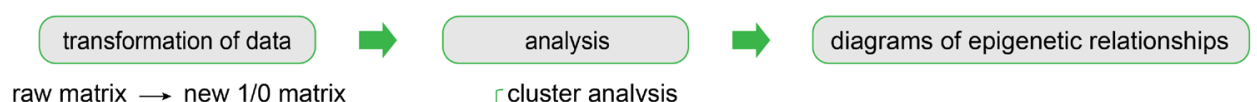

raw matrix $\rightarrow$ new $1 / 0$ matrix $\quad$ cluster analysis

Fig. 1. The methylation-sensitive amplified polymorphism analysis process was established for qualifying the methylation of different samples (the samples treated under one factor, such as tissues) with the same genotype at one level and at different levels (the levels belonging to another factor, such as a stage). A map with amplified bands was obtained via the digestion of DNA by EcoRI/HpaII (H) and EcoRI/MspI (M), ligation, PCR, and electrophoresis; the presence of amplified bands was recorded as 1, and the absence of that was recorded as 0 , which formed the $1 / 0$ matrix. Types I $(1,1)$, II $(1,0)$, and III $(0,1)$ refer to unmethylation, hemi-methylation, and full methylation, respectively; the status for type IV $(0,0)$ needs to be confirmed, and it indicates a methylation status (hypermethylation) when other samples with the same genotype present any one of the other three types at the same site. The methylation levels were obtained by calculating the proportions of different types of bands in the total bands. In the analysis of methylation patterns, the quantitative methods for the methylation events of the samples at one level and different levels were given. For analysis of the samples at one level, the methylation events were classified into the same and polymorphic patterns. The same patterns include two types: nonmethylation state preservation and no change in pattern of methylation state. Polymorphic patterns mean the different patterns among samples, and as a special form of polymorphic patterns, specific patterns were defined as the patterns in one sample different from the uniform patterns at the same sites of other samples, which reflects the methylation specificity on a certain sample, especially when compared with more samples (two or more samples). The specific patterns include three types: demethylation (DM), de novo 
methylation (DNM), and changes in pattern of methylation state (CMS). When there are three or more samples at one level for analysis, the specific patterns shown in 'sample 1' were just taken as examples, and in fact, there also exist sites with the specific patterns shown in other samples; in addition, the pairwise comparison and overall analysis of these samples can be performed meanwhile to fully understand the methylation changes among them. For analysis of the samples at different levels, the methylation events were classified into different types, including the same patterns present among the samples at any level, polymorphic patterns present among the samples at one level, two (or more) levels and any level. To reveal the epigenetic structure among the samples, the raw matrix should be first transformed into the final binary matrix, and then analytical methods, such as cluster analysis and principal coordinate analysis (PCoA), were performed. In this analysis process, the listed samples and levels were just used as examples for explanation of the analytical methods, and for more samples at one stage or more stages, the methods are also suitable.

In addition to the pairwise comparisons, the overall methylation status at the sites of the samples of each tissue at the three stages (IE, OSG, and FM) was analyzed. For the leaf or xylem samples collected at the three stages, the polymorphic patterns included completely different patterns and specific patterns. The polymorphism rates (the rate of sites with specific and completely different patterns) among the leaves at the three stages were 55.03 and $54.92 \%$ for 'Pawnee' and 'Stuart', respectively, and were lower than those among the xylems at the three stages (72.77 and $68.47 \%$ for 'Pawnee' and 'Stuart', respectively), which was in agreement with the smaller changes in the methylation status of the leaves compared with that in the xylems between any two stages for each cultivar. Among the specific rates (rates of the sites with specific patterns) for the leaves at the three stages, the specific rate for the leaf at the FM stage was the highest in each cultivar (23.16 and $22.26 \%$ for 'Pawnee' and 'Stuart', respectively). Different from the results obtained from the leaves, among the specific rates for the xylems at the three stages of 'Pawnee', the specific rate for the xylem at the OSG stage was the highest $(25.76 \%)$, which may be attributable to the highest variation frequency in methylation between the xylems at the IE and OSG stages of 'Pawnee' (Fig. 6).

Based on the 'mixing-scoring 2' approach, cluster analysis and PCoA were performed to explore the epigenetic distances and relationships among the six tissue samples at the three stages of two pecan cultivars. The Dice coefficients for the unmethylation matrix $(0.591-0.799)$ and hypermethylation matrix $(0.415-0.736)$ were higher

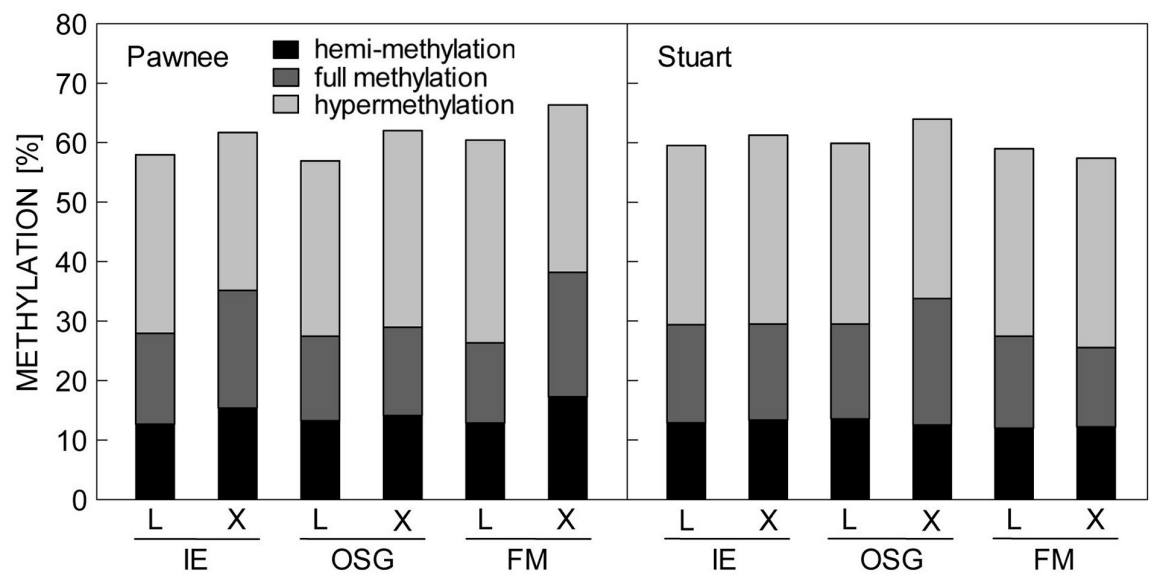

Fig. 2. The methylation levels of leaf (L) and xylem (X) at inflorescence emergence (IE), ovary start growth (OSG), and fruit maturity (FM) stages in pecan cultivars Pawnee and Stuart.

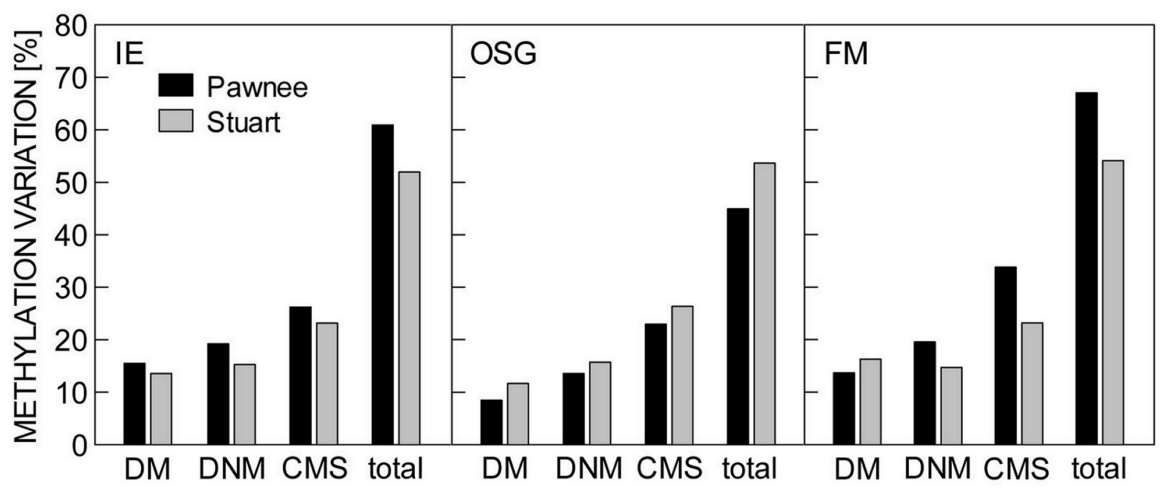

Fig. 3. The frequencies of methylation variation from the leaf to the xylem at inflorescence emergence (IE), ovary start growth (OSG), and fruit maturity (FM) stages in pecan cultivars Pawnee and Stuart (DM - demethylation, DNM - de novo methylation, CMS - changes in pattern of methylation state). 
than those for the hemi-methylation matrix $(0.183-0.423)$ and full methylation matrix $(0.243-0.453)$. The clustering results for the six tissue samples based on the four types of methylation matrixes were consistent overall. The xylem samples at the IE and FM stages were separated from the six tissue samples, and the closest methylation relationship was shown between the leaf samples at the IE and OSG stages. However, in the epigenetic relationships among the leaf (IE), leaf (OSG), leaf (FM) and xylem (OSG), there existed some different results from different types of methylation matrixes. According to the unmethylation and hypermethylation matrixes, the leaf (FM) had a closer epigenetic relationship with leaf (IE) and leaf (OSG) than did the xylem (OSG) with the two leaf samples, whereas different results were obtained based on the hemimethylation and full methylation matrixes (Fig. 2 Suppl.: $\mathrm{C} 1, \mathrm{D} 1, \mathrm{E} 1$, and F1). By the two-dimensional diagrams of the previous two principal coordinates (Fig. 2 Suppl.: C2, D2, E2, and F2), the epigenetic relationships among the tissue samples were also revealed intuitively, and the results from the PCoA were generally consistent with those of the cluster analysis.

\section{Discussion}

In this study, the F-MSAP technique was used to detect the methylation patterns in the leaves and xylems at three developmental stages of two pecan cultivars Pawnee and Stuart. Using the tissues with the same genotype from different stages as templates, we summarized and established the quantitative analysis of the MSAP data. The analytical methods in this process can be widely applied in the analysis of biological processes, such as comparison of control and stressed samples at different periods, which is also a cytosine methylation research topic that has received substantial attention. Notably, for samples with different genotypes (e.g., different cultivars) in which the detected type IV $(0,0)$ has an uncertain status (sequence mutations or hypermethylation) and is usually treated as missing data, the analytical methods in this process can also be used as a reference to obtain valuable information and key markers, although the full methylation information in the CCGG sites cannot be revealed.

According to the statistical analysis of the F-MSAP data obtained from the pecan tissues at different stages of the two cultivars, the total methylation was in the range of 56.95 to $66.33 \%$, which was higher than in most previously reported plants (no more than $50 \%$; Liu et al. 2018) for which the MSAP method was applied. In addition to the possible methylation difference among species, the statistical analysis of the type IV bands in this study may be an important factor triggering the higher methylation level because type IV $(0,0)$ has rarely been considered in previous methylation studies. Furthermore, F-MSAP has been shown to produce more and higher recognizable polymorphic fragments than the traditional MSAP method (Huang and Sun 1999), which may also explain this result. In this study, the total methylation in pecan xylem was generally higher than that in the leaf at each stage, which is consistent with the results that showed that the methylation in the developing xylem was higher than in young and mature leaves in Populus tomentosa (Wang et al. 2016). Previously, it was reported that in some plants, mature tissues have a higher methylation than younger tissues (Messeguer et al. 1991, Finnegan et al. 1998, Kaźmierczak 1998, Jia et al. 2011, Osabe et al. 2014). However, a lower DNA methylation was observed in rice flag leaves than in seedlings (Xiong et al. 1999). In this study, there was not a consistent changing trend with the developmental processes in either the leaf or xylem, and an opposite trend was even observed between the methylation in the leaf and xylem with the developmental process. A study on carrots also revealed different trends of methylation in different tissues/organs during the growth and the leaves methylation increases from seedling to adult plants, but decreases during root maturation (Palmgren et al. 1991). According to these studies, no absolute relationship is presented between the methylation level and the development. Notably, epigenetic factors other than DNA methylation, such as histone modification and small RNAs, can also modulate gene expression to affect development (Karim et al. 2016, Alonso et al. 2017), which may be a reason for the erratic changes in methylation during it.

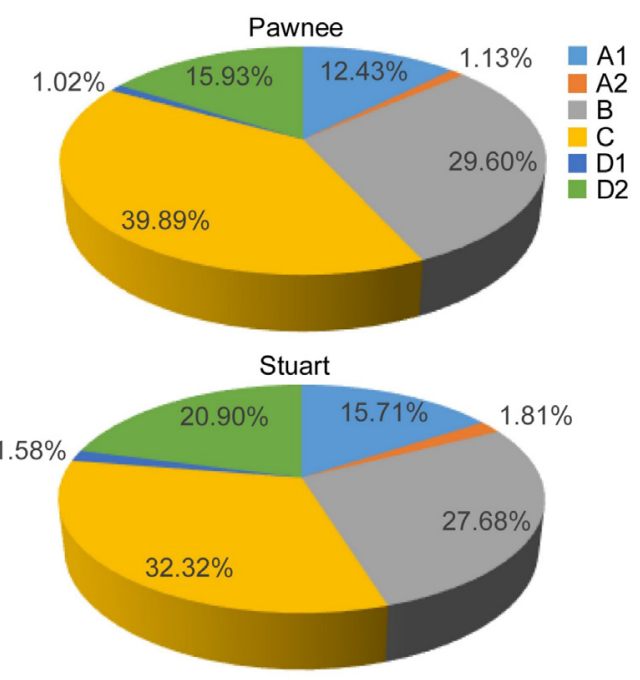

Fig. 4. Comparison of the methylation patterns in the leaf and xylem during inflorescence emergence, ovary start growth, and fruit maturity stages. The methylation events occurring between the leaf and the xylem during the three stages were classified into four classes: class A - the same patterns present between the two tissues at any stage (including A1 and A2; A1, a special form of class A, represents identical patterns present at all of the six tissue samples from three stages); class B - different patterns present between the two tissues at one stage; class $\mathrm{C}$ - different patterns present between the two tissues at two stages; class D - different patterns present between the two tissues at any stage (including D1 and D2; D1, a special form of class D, refers to the same different patterns present between the two tissues among the three stages, namely, the methylation events that patterns are the same in the leaves and xylem, respectively, at three stages, but they are different between leaves and xylem). The percentages represent the rates of the sites with these classes of methylation events. 
In contrast to the methylation that reflect the entire distribution of methylated sites among the CCGG sites, the differential methylation patterns directly reflect the information of methylation variation at the detailed sites and are often regarded as the focus of research. According to previous reports, different extents of bases or sites are differentially methylated in different plant tissues. For example, the DNA methylation differences were computed between the roots and shoots of Arabidopsis at single cytosines, and the results showed that one in every 173 cytosines is differentially methylated (Widman et al. 2014). A study on Populus trichocarpa showed that one third of the genome is differentially methylated among the tissues (Vining et al. 2012). The detection of the methylation patterns among the tissues of Populus tomentosa suggested that $26.81 \%$ of the sites present tissue- or organ-specific cytosine methylation (Wang et al. 2016). In this study, extensive differential methylation was found at the amplified sites of the leaves and xylems, with a range of 44.97 to $67.01 \%$ for the overall variation frequencies (sum of $\% \mathrm{DM}, \% \mathrm{DNM}$ and $\% \mathrm{CMS}$ ) between the two tissues at the three stages of the two cultivars. By pairwise comparison and overall analysis of each tissue at the three stages, this study quantified the methylation differences and tracked the dynamics in the leaf and xylem across development. The results showed that the total variation frequencies in methylation between the leaves at any two stages were $31.86-45.88 \%$, and 40.90 - $59.44 \%$ between the xylems. The polymorphism rates of methylation among the leaves at the three stages were 55.03 and $54.92 \%$ for 'Pawnee' and 'Stuart', respectively, with polymorphism rates of 72.77 and $68.47 \%$ for the xylems of 'Pawnee' and 'Stuart', respectively. Similar to the extensive variation in the sites of different tissues, these data also showed a wealth of methylation changes among the samples of each tissue at different developmental stages. The relatively high percentages of methylation variation between tissues or stages may be partly because of the different methods used for methylation detection. The MSAP data were obtained based on amplification with primers. Compared with other methods, such as bisulfite sequencing and methylated DNA immunoprecipitation sequencing (MeDIP-Seq), the detection range within the genome of MSAP is not so wide. Therefore, this factor may affect the obtained percentages of differentially methylated sites between tissues or stages. In addition, methylation changes with differentiation and development may result from the different development characteristics and growth environments of plants. Plant development is plastic and influenced by multiple environmental and endogenous cues (Valledor et al. 2007, Viejo et al. 2012). Hence, plants require a specific interaction between developmental programmes and signalling pathways triggered from external stimuli that are coordinated at the level of chromatin organization (Reyes et al. 2002). As one type of epigenetic change, DNA methylation can be involved in controlling the functional state of the chromatin and gene expression that underpins the reprogramming of cells necessary for plant development (Viejo et al. 2012). Pecan, as a woody tree, undergoes long-term development and perennial exposure to environmental conditions, which is different from the lifecycle of annual plants. Therefore, in the differentiation and development of the leaf and xylem of pecan, the rearrangement of methylation patterns at a wide range of amplified sites may be needed in response to the demand of endogenous development and environmental stimuli.

The regulation of methylation or DM on gene expression has been well studied. Usually, methylation in the gene promoter inhibits gene expression, while there are

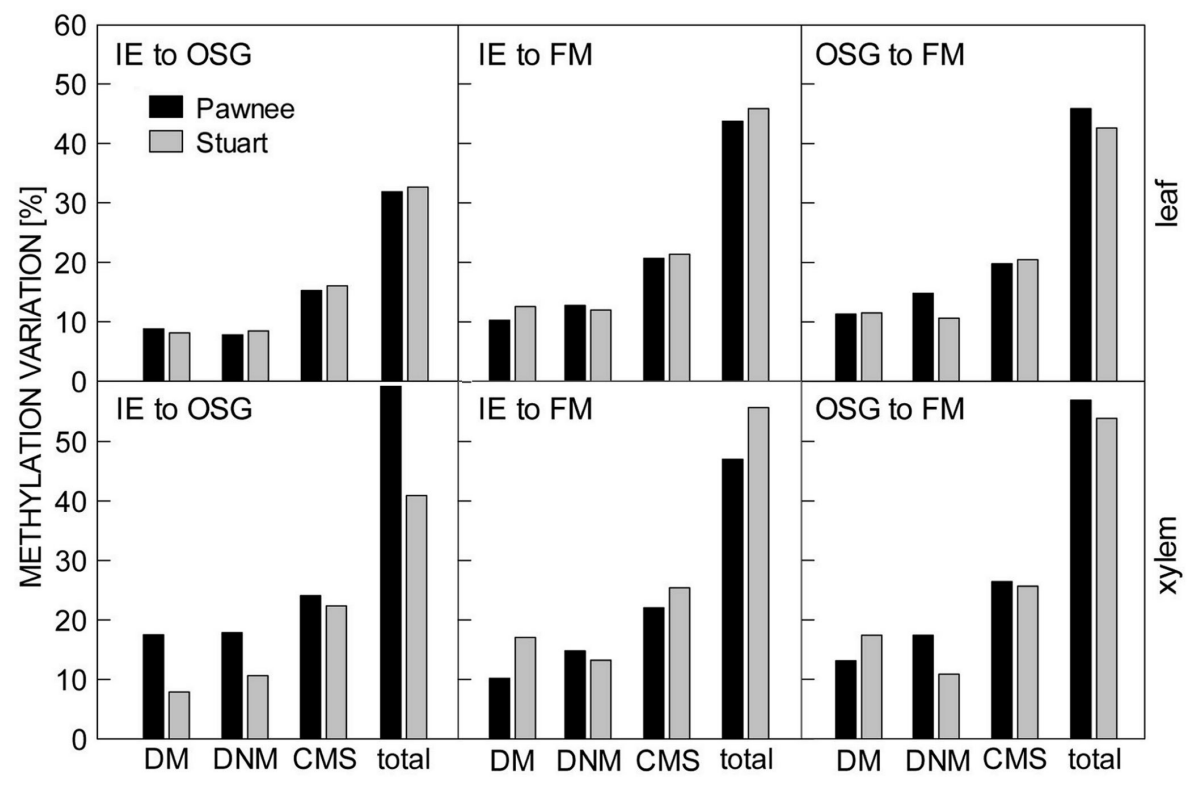

Fig. 5. The frequencies of methylation variation between inflorescence emergence (IE), ovary start growth (OSG), and fruit maturity (FM) stages of the leaf and xylem in two cultivars (Pawnee and Stuart). DM - demethylation, DNM - de novo methylation, CMS - changes in pattern of methylation state. 
different effects or no effects of methylation in the gene body on gene expression (Zhang et al. 2006, Vining et al. 2012, Candaele et al. 2014). However, recent studies have suggested that methylation in different sequence contexts also has different effects on gene expression. Roessler et al. (2016) found that differential CG methylation consistently covaried negatively with gene expression, while either $\mathrm{CHG}$ or $\mathrm{CHH}$ methylation in different regions showed less consistent relationships with gene expression. Furthermore, as the MSAP study on poplar showed, hemi-methylation and full methylation tend to inhibit gene transcription, but the amount of transcripts of hemi-methylated genes are significantly lower than those of the fully methylated genes (Wang et al. 2016). With the effects on gene expression, the detected methylation variations (including the types of DM, DNM and CMS) in this study implied the important roles of methylation in the regulation of tissue differentiation and development in pecan trees. Notably, to further learn about the methylation variation between the leaf and xylem in the same sites of the tissues of three stages, the quantitative method was creatively conducted in this study for the overall analysis of the methylation variation between the tissues at the three stages. In this analytical method, the methylation events were classified into four classes of methylation patterns (the different patterns present between two tissues at no stage, one stage, two stages, and any stage class), and it was observed that the sites with class $\mathrm{C}$ (different patterns present between two tissues at two stages) were at the highest proportion, 39.89 and $32.32 \%$ for 'Pawnee' and 'Stuart', respectively. This process also reveals the ample methylation variation existing between the leaf and xylem from another perspective. As shown by previous studies, markers with differential methylation patterns can be obtained by PAGE and sequenced for research on the effects of methylation on gene expression and their regulatory functions (Zhang et al. 2011, Ci et al. 2016, Wang et al. 2016). In this study, 1.02 and $1.58 \%$ (in 'Pawnee' and 'Stuart', respectively) of all detected sites showed the same specific patterns between the leaf and xylem among the three stages, and these sites may be significant markers involved in the differentiation of the
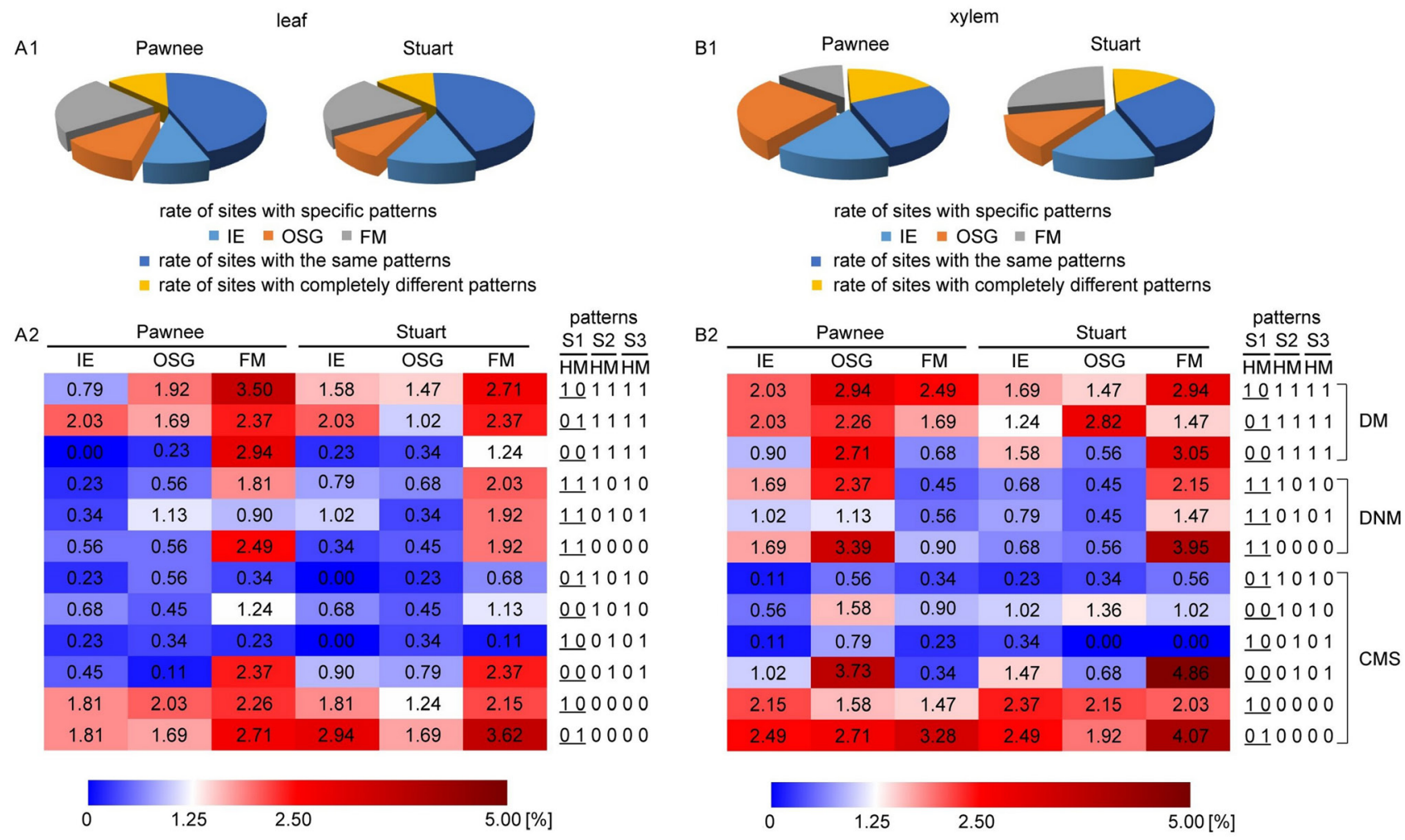

Fig. 6. Overall analysis of the methylation status of the samples of each tissue (leaf and xylem) at inflorescence emergence (IE), ovary start growth (OSG), and fruit maturity (FM) stages of two pecan cultivars (Pawnee and Stuart). The methylation patterns can be classified into three classes: 1) the same patterns present in the samples of each tissue at the three stages; 2) specific patterns present in the samples of each tissue at the IE, OSG, and FM stages, respectively; and 3) completely different patterns in the samples of each tissue at the three stages. The rates of the sites with these types of patterns are shown in $A 1$ (leaf) and B1 (xylem). $A 2$ (leaf) and $B 2$ (xylem) show the rates of the sites with the detailed specific patterns present in the tissue samples at the three stages. 1 and $O$ refer to the presence and absence, respectively, of the fragments generated by double digestion with $E c o \mathrm{RI} / H p a \mathrm{II}(\mathrm{H})$ or $E c o \mathrm{RI} / M s p \mathrm{I}(\mathrm{M})$. The $(1,1)$, (1, 0), $(0,1)$, and $(0,0)$ refer to unmethylation, hemi-methylation, full methylation, and hypermethylation, respectively. The S1, S2, and S3 represent the three samples of each tissue at the three stages, and the data in the heat map represent the rate of the sites with the specific patterns underlined for the tissue at the certain stage. The specific patterns were defined as the patterns in one sample that were different from the uniform patterns at the same sites of other samples including three types: demethylation (DM), de novo methylation (DNM), and changes in the pattern of methylation state (CMS). 
two tissues. Thus, it appears meaningful to conduct more in-depth studies on these candidate markers in the future.

In addition to the investigation of the differences in the methylation patterns among different tissues and stages, this study was also designed to explore the changes in methylation differences between the leaf and xylem across stages and the differences between the leaf and xylem in methylation variation among different stages, which has been nearly unreported in other species. The results showed that for both cultivars, the total variation frequency in methylation status between the leaf and xylem was highest at the FM stage. DNA methylation has unique dynamics during specific developmental stages (Valledor et al. 2007). A study on tobacco also indicated that marked changes in the methylation status occurs with the transition from the vegetative to the reproductive stage (Zhang et al. 2015). For pecan trees, the leaves and xylem developed relatively fast at the IE and OSG stages, and vegetative growth became quite slow at FM. Therefore, at the FM stage, specific changes in the methylation patterns possibly occurred at either or both the leaf and xylem, which resulted in the largest methylation differences between the leaf and xylem at this stage. According to the analysis of specific patterns for the leaf or xylem samples at the three stages, it was observed that the specific rate for the FM stage was the highest in the leaves of each cultivar, which at least supports that more specific methylation changes exist in the leaves at the FM stage. This study also found that the leaf showed smaller changes than the xylem in methylation status between any two stages, suggesting that the methylation patterns in the leaf may be more stable than those in the xylem. In addition, based on the higher polymorphism rate present among the xylems from the three stages than that among the leaves for each cultivar, it was speculated that more genes may be involved in the development of the xylem than in the leaves.

To study the epigenetic structure and relationships based on MSAP profiles, different scoring methods are used (Schulz et al. 2013). In recent years, the 'mixed scoring 2' approach has been applied frequently (Schulz et al. 2014, Gáspár et al. 2019), although it was proposed later. Given the possibly different regulatory roles of the different methylation patterns, this approach separately takes into account different methylation types and can draw more detailed information on epigenetic variation. By reference to this approach, we recoded the raw matrix into four separate matrixes corresponding to the four patterns (unmethylation, hemi-methylation, full methylation, and hypermethylation). Through the cluster analysis and PCoA of the four matrixes, it was commonly obtained that the xylem samples at the IE and FM stages were separated from the six tissue samples, which suggested that they had relatively distant epigenetic relationships with other tissue samples in the whole. In addition, the epigenetic relationships among the tissue samples established based on the unmethylation and hypermethylation matrixes were substantially closer than those based on the hemimethylation and full methylation matrixes. It can be concluded that in the process of tissue differentiation and development, the methylation variation involved in the hemi-methylation and full methylation states was ampler than the variation in the unmethylation and hypermethylation patterns.

\section{References}

Alonso, C., Medrano, M., Pérez, R., Bazaga, P., Herrera, C.: Tissue-specific response to experimental demethylation at seed germination in the non-model herb Erodium cicutarium. - Epigenomes 1: 16, 2017.

An, Y.C., Goettel W., Han Q., Bartels A., Liu Z., Xiao W.: Dynamic changes of genome-wide DNA methylation during soybean seed development. - Sci. Rep. 7: 12263, 2017.

Bednarek, P.T., Orłowska, R., Niedziela, A.: A relative quantitative methylation-sensitive amplified polymorphism (MSAP) method for the analysis of abiotic stress. - BMC Plant Biol. 17: 79, 2017.

Candaele, J., Demuynck, K., Mosoti, D., Beemster, G.T., Inzé, D., Nelissen, H.: Differential methylation during maize leaf growth targets developmentally regulated genes. - Plant Physiol. 164: 1350-1364, 2014.

Cao, X., Jacobsen, S.E.: Role of the Arabidopsis DRM methyltransferases in de novo DNA methylation and gene silencing. - Curr. Biol. 12: 1138-1144, 2002.

Ci, D., Song, Y., Du, Q., Tian, M., Han, S., Zhang, D.: Variation in genomic methylation in natural populations of Populus simonii is associated with leaf shape and photosynthetic traits. - J. exp. Bot. 67: 723-737, 2016.

Finnegan, E.J., Genger, R.K., Peacock, W.J., Dennis, E.S.: DNA methylation in plants. - Annu. Rev. Plant Physiol. Plant mol. Biol. 49: 223-247, 1998.

Finnegan, E.J., Peacock, W.J., Dennis, E.S.: Reduced DNA methylation in Arabidopsis thaliana results in abnormal plant development. - Proc. nat. Acad. Sci. USA 93: 8449-8454, 1996.

Gáspár, B., Bossdorf, O., Durka, W.: Structure, stability and ecological significance of natural epigenetic variation: a large-scale survey in Plantago lanceolata. - New Phytol. 221: 1585-1596, 2019.

Han, M., Peng, F., Marshall, P.: Pecan phenology in Southeastern China. - Ann. appl. Biol. 172:160-169, 2018.

He, X.J., Chen, T., Zhu, J.K.: Regulation and function of DNA methylation in plants and animals. - Cell Res. 21: 442, 2011.

Herrera, C.M., Bazaga, P.: Epigenetic differentiation and relationship to adaptive genetic divergence in discrete populations of the violet Viola cazorlensis. - New Phytol. 187: 867-876, 2010.

Huang, J., Sun, M.: A modified AFLP with fluorescence-labelled primers and automated DNA sequencer detection for efficient fingerprinting analysis in plants. - Biotechnol. Tech. 13: 277278, 1999.

Inácio, V., Barros, P.M., Costa, A., Roussado, C., Gonçalves, E., Costa, R., Graça, J., Oliveira, M.M., Morais-Cecílio, L.: Differential DNA methylation patterns are related to phellogen origin and quality of Quercus suber Cork. - PLoS ONE 12: e0169018, 2017.

Jia, F., Fu, Y., Liu, W., Zhao, Y.: Quantitative determination of DNA methylation in tobacco leaves by HPLC. - Afr. J. aAgr. Res. 6: 1545-1548, 2011.

Jullien, P.E., Kinoshita T., Ohad N., Berger F.: Maintenance of DNA methylation during the Arabidopsis life cycle is essential for parental imprinting. - Plant Cell 18: 1360-1372, 2006.

Karim, R., Nuruzzaman, M., Khalid, N., Harikrishna, J.: Importance of DNA and histone methylation in in vitro plant 
propagation for crop improvement: a review. - Ann. appl. Biol. 169: 1-16, 2016

Kaźmierczak, J.: Effect of DNA methylation on potential transcriptional activity in different tissues and organs of Vicia faba ssp. minor.. - Folia histochem. cytobiol. 36: 45-49, 1998.

Law, J.A., Jacobsen S.E.: Establishing, maintaining and modifying DNA methylation patterns in plants and animals. - Nat. Rev. Genet. 11: 204-220, 2010.

Li, Q., Gent, J.I., Zynda, G., Song, J.W., Makarevitch, I., Hirsch, C.D., Hirsch, C.N., Dawe, R.K., Madzima, T.F., McGinnis, K.M., Lisch, D., Schmitz, R.J., Vaughn, M.W., Springer, N.M.: RNA-directed DNA methylation enforces boundaries between heterochromatin and euchromatin in the maize genome. - Proc. nat. Acad. Sci. USA 112: 14728-14733, 2015.

Li, Y., Zhao, F., Yang, X., Zhong, S., Li, F., Lin, C., Wu, H., Guo, W., Liao, F.: Cytosine methylation analysis of Pinus elliottii $\times$ Pinus caribaea var. hondurensis and their parental lines. - J. Plant Growth Regul. 38: 30-38, 2018.

Liu, Z., Chen, T., Peng, F., Liang, Y., Tan, P., Mo, Z., Cao, F., Shang, Y., Zhang, R., Li, Y.: Variation in cytosine methylation among pecan cultivars at different developmental stages. - J. amer. Soc. hort. Sci. 143: 173-183, 2018.

Lombardini, L., Restrepo-Diaz, H., Volder, A.: Photosynthetic light response and epidermal characteristics of sun and shade pecan leaves. - J. amer. Soc. hort. Sci. 134: 372-378, 2009.

Lu, Y., Rong, T., Cao, M.: Analysis of DNA methylation in different maize tissues. - J. Genet. Genomics 35: 41-48, 2008.

Matzke, M.A., Mosher, R.A.: RNA-directed DNA methylation: an epigenetic pathway of increasing complexity. - Nat. Rev. Genet. 15: 394, 2014

Messeguer, R., Ganal, M.W., Steffens, J.C., Tanksley, S.D.: Characterization of the level, target sites and inheritance of cytosine methylation in tomato nuclear DNA. - Plant mol. Biol. 16: 753-770, 1991.

Osabe, K., Clement, J.D., Bedon, F., Pettolino, F.A., Ziolkowski, L., Llewellyn, D.J., Finnegan, E.J., Wilson, I.W.: Genetic and DNA methylation changes in cotton (Gossypium) genotypes and tissues. - PLoS ONE 9: e86049, 2014.

Palmgren, G., Mattsson, O., Okkels, F.T.: Specific levels of DNA methylation in various tissues, cell lines, and cell types of Daucus carota. - Plant Physiol. 95: 174-178, 1991.

Reyes, J.C., Hennig, L., Gruissem, W.: Chromatin-remodeling and memory factors. New regulators of plant development. Plant Physiol. 130: 1090-1101, 2002.

Reyna-López, G.E., Simpson, J., Ruiz-Herrera, J.: Differences in DNA methylation patterns are detectable during the dimorphic transition of fungi by amplification of restriction polymorphisms. - Mol. Genet. Genomics 253: 703-710, 1997.

Roessler, K., Takuno, S., Gaut, B.S.: CG methylation covaries with differential gene expression between leaf and floral bud tissues of Brachypodium distachyon. - PLoS ONE 11: e0150002, 2016

Salmon, A., Clotault, J., Jenczewski, E., Chable, V., ManzanaresDauleux, M.J.: Brassica oleracea displays a high level of DNA methylation polymorphism. - Plant Sci. 174: 61-70, 2008.

Schulz, B., Eckstein, R.L., Durka, W.: Scoring and analysis of methylation-sensitive amplification polymorphisms for epigenetic population studies. - Mol. Ecol. Resour. 13: 642653, 2013.

Schulz, B., Eckstein, R.L., Durka, W.: Epigenetic variation reflects dynamic habitat conditions in a rare floodplain herb. Mol. Ecol. 23: 3523-3537, 2014.

Soppe, W.J.J., Jasencakova, Z., Houben, A., Kakutani, T., Meister, A., Huang, M.S., Jacobsen, S.E., Schubert, I., Fransz, P.F.: DNA methylation controls histone H3 lysine 9 methylation and heterochromatin assembly in Arabidopsis. - EMBO J. 21: 6549-6559, 2002.

Thompson, T.E., Hunter R.E.: 'Pawnee' pecan. - Hortscience 20 776, 1985

Valledor, L., Hasbún, R., Meijón, M., Rodríguez, J.L., Santamaría, E., Viejo, M., Berdasco, M., Feito, I., Fraga, M.F., Cañal, M.J., Rodríguez, R.: Involvement of DNA methylation in tree development and micropropagation. - Plant Cell Tissue Organ Cult. 91: 75-86, 2007.

Vaughn, M.W., Tanurdžić, M., Lippman, Z., Jiang, H., Carrasquillo, R., Rabinowicz, P.D. Dedhia, N., McCombie, W.R., Agier, N., Bulski, A., Colot, V., Doerge, R.W., Martienssen, R.A.: Epigenetic natural variation in Arabidopsis thaliana. - PLoS Biol. 5: e174, 2007.

Viejo, M., Santamaría, M.E., Rodríguez, J.L., Valledor, L., Meijón, M., Pérez, M., Pascual, J., Hasbún, R., Fernández Fraga, M., Berdasco, M., Toorop, P.E., Cañal, M.J., Rodríguez Fernández, R.: Epigenetics, the role of DNA methylation in tree development. - In: Loyola-Vargas V., Ochoa-Alejo N. (ed.): Plant Cell Culture Protocols. Methods in Molecular Biology. Pp. 277-301. Humana Press, Totowa 2012.

Vining, K.J., Pomraning, K.R., Wilhelm, L.J., Priest, H.D., Pellegrini, M., Mockler, T.C., Freitag, M., Strauss, S.H.: Dynamic DNA cytosine methylation in the Populus trichocarpa genome: tissue-level variation and relationship to gene expression. - BMC Genomics 13: 27, 2012.

Wang, Q., Ci, D., Li, T., Li, P., Song, Y., Chen, J., Quan, M., Zhou, D., Zhang, D.: The role of DNA methylation in xylogenesis in different tissues of poplar. - Front. Plant Sci. 7:1003, 2016.

Wang, W.S., Pan, Y.J., Zhao, X.Q., Dwivedi, D., Zhu, L.H., Ali, J., Fu, B.Y., Li, Z.K.: Drought-induced site-specific DNA methylation and its association with drought tolerance in rice (Oryza sativa L.). - J. exp. Bot. 62: 1951-1960, 2011.

Widman, N., Feng, S., Jacobsen, S.E., Pellegrini, M.: Epigenetic differences between shoots and roots in Arabidopsis reveals tissue-specific regulation. - Epigenetics 9: 236-242, 2014.

Xiong, L.Z., Xu, C.G., Saghai Maroof, M.A., Zhang, Q.: Patterns of cytosine methylation in an elite rice hybrid and its parental lines, detected by a methylation-sensitive amplification polymorphism technique. - Mol. gen. Genet. 261: 439-446, 1999.

Xu, Q., Sun, D., Zhang, Y.: F-MSAP: a practical system to detect methylation in chicken genome. - Chin. Sci. Bul. 50: 20392044,2005

Zhang, H., Lang, Z., Zhu, J.K. Dynamics and function of DNA methylation in plants. - Nat. Rev. mol. cell. Biol. 19: 489-506, 2018.

Zhang, M., Kimatu, J.N., Xu, K., Liu, B.: DNA cytosine methylation in plant development. - J. Genet. Genomics 37: $1-12,2010$.

Zhang, M., Xu, C., Von Wettstein, D., Liu, B.: Tissue-specific differences in cytosine methylation and their association with differential gene expression in sorghum. - Plant Physiol. 156: 1955-1966, 2011.

Zhang, P.Y., Wang, J.G., Geng, Y.P., Dai, J.R., Zhong, Y., Chen, Z.Z., Zhu, K., Wang, X.Z., Chen, S.Y.: MSAP-based analysis of DNA methylation diversity in tobacco exposed to different environments and at different development phases. - Biochem. Syst. Ecol. 62: 249-260, 2015

Zhang, X., Yazaki, J., Sundaresan, A., Cokus, S., Chan, S.W., Chen, H., Henderson, I.R., Shinn, P., Pellegrini, M., Jacobsen, S.E., Ecker, J.R.: Genome-wide high-resolution mapping and functional analysis of DNA methylation in Arabidopsis. - Cell 126: 1189-1201, 2006.

Zhao, Y., Chen, M., Storey, K.B., Sun, L., Yang, H.: DNA methylation levels analysis in four tissues of sea cucumber 
Apostichopus japonicus based on fluorescence-labeled methylation-sensitive amplified polymorphism (F-MSAP) during aestivation. - Comp. Biochem. Physiol. B Biochem. mol. Biol. 181: 26-32, 2015.

Zhong, S., Fei, Z., Chen, Y., Zheng, Y., Huang, M., Vrebalov,
J., McQuinn, R., Gapper, N., Liu, B., Xiang, J., Shao, Y., Giovannoni, J.J.: Single-base resolution methylomes of tomato fruit development reveal epigenome modifications associated with ripening. - Natur. Biotechnol. 31: 154-159, 2013. 